Comment. Math. Helv. 75 (2000) 594-607

(C) 2000 Birkhäuser Verlag, Basel

0010-2571/00/040594-14\$1.50+0.20/0

Commentarii Mathematici Helvetici

\title{
Asymptotic degeneration of representations of quivers
}

Volker Strassen

\begin{abstract}
We define asymptotic degeneration of nilpotent representations of an arbitrary finite quiver, using large tensor powers and small direct sums, and characterize this notion by a simple and effective criterion.
\end{abstract}

Mathematics Subject Classification (2000). 16G20, 06F25.

Keywords. Quiver, representation, degeneration, tensor product, asymptotics.

\section{Introduction}

We start with a finite quiver $Q$ and study degenerations of $Q$-modules, i.e. of finite dimensional representations of $Q$ over $\mathbb{C}$, in the sense of orbit closure. (See [9], [3], [7], [2]; [1], [15] for definitions.) We write $U \unlhd V$ to express the fact that $U$ is a degeneration of $V .^{1}$

Building on work of Abeasis and Del Fra [1] and Riedtmann [15], Bongartz [4], [5] has given practicable necessary and sufficient conditions for degeneration when $Q$ is a tame quiver, i.e., when the underlying undirected graph is an extended Dynkin diagram. There seems to be little hope for results of a similar quality in the case of wild quivers (in spite of Zwara's recent breakthrough [18]).

Let $Q$ be a finite quiver, $Q_{0}$ its sets of points and $Q_{1}$ its set of arrows. A $Q$ module $T$ is called trivial, when $T(l)=0$ for all $l \in Q_{1}$. We call $Q$-modules $U$ and $V$ equivalent, when there exist trivial modules $S$ and $T$ such that $U \oplus S \simeq V \oplus T$. The $\operatorname{set}^{2} \mathcal{M}(Q)$ of equivalence classes of $Q$-modules is a commutative semiring with unit element, where addition and multiplication are induced by direct sum and tensor product of modules. (The tensor product of $U$ and $V$ is defined by $(U \otimes V)(\alpha):=U(\alpha) \otimes V(\alpha)$ for $\alpha \in Q_{0}$ and $(U \otimes V)(l):=U(l) \otimes V(l)$ for $\left.l \in Q_{1}.\right)^{3}$ Degeneration of modules induces a partial order in $\mathcal{M}(Q)$ :

$$
p \unlhd q: \Longleftrightarrow \exists U \in p, V \in q \quad U \unlhd V .
$$

This partial order is compatible with addition and multiplication in the sense that $p \unlhd q$ implies $p+r \unlhd q+r$ and $p \cdot r \unlhd q \cdot r$ for any $p, q, r \in \mathcal{M}(Q)$. In short: $\mathcal{M}(Q)$ is an ordered commutative semiring with unit element. ${ }^{4}$ 
A $Q$-module $V$ is nilpotent, when there exists a positive integer $\nu$ such that $V(w)=0$ for all paths $w$ of length at least $\nu$. This is preserved under equivalence. Every $Q$-module is nilpotent unless $Q$ contains directed cycles. The trivial modules are exactly the semisimple nilpotent modules. Submodules, homomorphic images, direct sums and tensor products of nilpotent modules are again nilpotent. In fact, the tensor product of a nilpotent module with an arbitrary $Q$-module is nilpotent. Therefore the set $\mathcal{N}(Q)$ of equivalence classes of nilpotent $Q$-modules is an ideal of the semiring $\mathcal{M}(Q)$. In particular $\mathcal{N}(Q)$ is an ordered commutative semiring, possibly lacking a unit element. An important additional property of $\mathcal{N}(Q)$ is $0 \unlhd q$ for all $q \in \mathcal{N}(Q) .^{5}$

First example: Consider the Jordan quiver $Q$ consisting of a single point $\alpha$ together with a single loop $l$. A nilpotent $Q$-module $V$ consists of a finite dimensional vector space $V(\alpha)$ together with a nilpotent linear endomorphimsm $V(l)$ of $V(\alpha)$. $U$ is a degeneration of $V$ when the closure of the conjugacy class of $V(l)$ contains an endomorphism isomorphic to $U(l)$. The semiring $\mathcal{N}(Q)$ can be identified with the semiring of functions $f: \mathbb{Z}_{>0} \rightarrow \mathbb{Z}_{>0}$ which have finite support and are convex in the sense that $f(j-1)+f(j+1)-2 f(j) \geq 0$ holds for all $j>1$. The isomorphism is given by $[V] \mapsto\left(j \mapsto R\left(V(l)^{j}\right)\right)$, where $[V]$ denotes the equivalence class of $V$ and $R$ denotes the rank function for linear maps between finite dimensional vector spaces. ${ }^{6}$ By a result of Gerstenhaber [11] (see also Kraft-Procesi [14]) the partial order $\unlhd$ in $\mathcal{N}(Q)$ becomes, under the above identification, the pointwise order of functions.

Second example: Let $Q$ be the quiver with $Q_{0}:=\{1, \ldots, n\}$ and $Q_{1}:=\{(i, i+$ 1) $: 1 \leq i<n\}$, i.e., the quiver of type $A_{n}$ with all arrows pointing in the same direction. Then $\mathcal{N}(Q)=\mathcal{M}(Q)$ may be identified with the semiring of interval functions $f:\{(i, j): 1 \leq i<j \leq n\} \rightarrow \mathbb{Z}_{\geq 0}$, satisfying $f(i-1, j) \leq f(i, j)$, $f(i, j+1) \leq f(i, j)$ and $f(i-1, j)+f(i, j+1) \leq f(i, j)+f(i-1, j+1)$, whenever these inequalities are meaningful. The isomorphism is given by $[V] \mapsto((i, j) \mapsto$ $R\left(V\left(w_{i, j}\right)\right)$ ), where $w_{i, j}$ denotes the unique directed path from $i$ to $j$. By a result of Abeasis and Del Fra [1] the partial order $\unlhd$ in $\mathcal{N}(Q)$ becomes, under the given identification, the pointwise order of interval functions.

Unfortunately, such neat descriptions of the ordered semiring $\mathcal{N}(Q)$ are rare. (See Corollary 2.) In the spirit of [17] we therefore introduce asymptotic degeneration $\gtrsim$ : For $p, q \in \mathcal{N}(Q)$ we set

$$
p \unlhd q: \Longleftrightarrow \exists k \in \mathbb{N} \forall N \gg 1 \quad p^{N} \unlhd N^{k} q^{N},
$$

where ' $\forall N \gg 1$ ' is a shorthand for ' $\exists N_{0} \forall N \geq N_{0}$ '. It is easy to see that $\triangleleft$ is a preorder in $\mathcal{N}(Q)$ compatible with multiplication. It is also compatible with addition, as follows, e.g., from Theorem 1 below. Of course $\unlhd$ implies $\triangleleft$. In the two examples above $\unlhd$ and $\triangleleft$ coincide, since here $(\mathcal{N}(Q), \unlhd)$ is isomorphic to a semiring of functions with pointwise order.

The following result shows that asymptotic degeneration can be characterized in a simple way. Recall that $R$ denotes the rank function for linear maps. By a path in $Q$ we always mean a directed path. 
Theorem 1. Let $Q$ be a quiver, $p, q \in \mathcal{N}(Q)$ and $U \in p, V \in q$. Then $p \triangleleft q$ if and only if the following two conditions are satisfied:

1. $R(U(w)) \leq R(V(w))$ for any path $w$ of positive length.

2. $U(v)=\theta \cdot U(w)$ whenever $\theta \in \mathbb{C}$ and $v, w$ are paths of positive length with the same starting point and the same end point and such that $V(v)=\theta \cdot V(w)$.

Theorem 1 will be proved in Section 3. (The necessity of the conditions is easy to see.)

Let us call a quiver $Q$ economical (sparsam), if for any $\alpha, \beta \in Q_{0}$ there is at most one path from $\alpha$ to $\beta$. Such a quiver does not contain directed cycles, hence $\mathcal{N}(Q)=\mathcal{M}(Q)$. Take an economical quiver $Q$. We define asymptotic equivalence of classes $p, q \in \mathcal{M}(Q)$ by

$$
p \sim q: \Longleftrightarrow p \unlhd q \& q \unlhd p
$$

Then $\sim$ is a congruence relation in $\mathcal{M}(Q)$ and therefore $\mathcal{M}(Q) / \sim$ is an ordered commutative semiring with unit element, the order being induced by asymptotic degeneration. Let $\Delta(Q)$ be the (finite) set of paths of $Q$ of positive length and let $\mathcal{F}(\Delta(Q), \mathbb{N})$ be the semiring of nonnegative integral valued functions on $\Delta(Q)$. We have a map

$$
\varphi: \mathcal{M}(Q) / \sim \mathcal{F}(\Delta(Q), \mathbb{N})
$$

which sends the asymptotic equivalence class of $[V] \in \mathcal{M}(Q)$ to the function $w \mapsto R(V(w))$.

Corollary 1. Let $Q$ be economical. Then $\varphi$ is an imbedding of ordered semirings.

Borrowing the language of [17], we may call $\Delta(Q)$ an asymptotic spectrum of $\mathcal{M}(Q){ }^{7}$

An interesting problem consists in characterizing the image of $\varphi$ in Corollary 1 in a way similar to its characterization in the special case of example 2.

The quiver of example 1 is not economical. Nevertheless, Corollary 1 holds in this case. This indicates that we have not stated the corollary in its most general form.

Corollary 2. (Riedtmann [16]) The connected quivers $Q$ for which $\unlhd$ and $₫$ coincide on $\mathcal{N}(Q)$ are the directed paths and the directed cycles.

Proof. The coincidence of $\unlhd$ and $\triangleleft$ for directed paths is the content of the second example above; the coincidence for directed cycles follows by generalizing the first example.

Conversely, suppose that $\unlhd$ and $₫$ coincide for the connected quiver $Q$. It suffices to show that $Q$ does not contain a subquiver isomorphic to either $\stackrel{1}{\longrightarrow} \bullet^{2} \longleftarrow^{3}$ or $\stackrel{1}{\bullet} \stackrel{2}{\bullet} \stackrel{3}{\bullet}$. By contradiction, suppose that $\stackrel{1}{\bullet} \longleftarrow \stackrel{2}{\bullet} \longrightarrow \stackrel{3}{\bullet}$ is a subquiver of $Q$. 
Let $U_{1}, U_{2}$ and $V$ be the indecomposable modules of $Q$ with supports $\{1,2\},\{2,3\}$ and $\{1,2,3\}$, respectively. Let $p$ and $q$ be the equivalence classes of $U:=U_{1} \oplus U_{2}$ and $V$, respectively. Then $p, q \in \mathcal{N}(Q)$ and by Theorem 1 we have $p \unlhd q$ and $q \unlhd p$, hence $p \unlhd q$ and $q \unlhd p$ by assumption, hence $p=q$, hence $U \oplus S \simeq V \oplus T$ for certain trivial modules $S, T$. This contradicts Krull-Schmidt.

Another way of phrasing Corollary 2 is that the connected quivers $Q$, for which $(\mathcal{N}(Q), \unlhd)$ is isomorphic to a semiring of functions with pointwise order, are the directed paths and the directed cycles.

\section{A Generalization}

The exposition of the proof of Theorem 1 will profit from the following generalization. Let $A$ be a finite dimensional associative $\mathbb{C}$-algebra with a multiplicative basis. This means that $A$ has a distinguished basis $B$ such that the product of any two elements of $B$ is either 0 or again an element of $B$ and such that $\operatorname{rad} A=\operatorname{lin} B_{1}$ for some $B_{1} \subset B$. Note that the unit element of $A$ does not necessarily belong to $B$.

By an $A$-module we mean a finite dimensional complex vector space together with a multiplicative linear map $A \rightarrow \operatorname{End}_{\mathbb{C}} V, a \mapsto a_{V}$. We do not assume $1_{V}=\mathrm{id}_{V}$, i.e. we allow nonunital modules. Clearly $1_{V}$ is a projection and a module endomorphism and therefore produces a direct decomposition of $V$ into the unital submodule $V_{u}:=\operatorname{im}\left(1_{V}\right)$ and the submodule $V_{0}:=\operatorname{ker}\left(1_{V}\right)$, which is annihilated by $A$. This decomposition is functorial.

We may and will identify an $A$-module structure on the vector space $V$ with its restriction to the basis $B$. Then such a structure is given by a map $B \rightarrow \operatorname{End}_{\mathbb{C}} V$, $b \mapsto b_{V}$ such that $b_{V} c_{V}=(b \cdot c)_{V}$ whenever $b \cdot c \in B$, and $b_{V} c_{V}=0$ otherwise. This map will be called the representation map of the module.

Besides the direct sum of two modules $U$ and $V$ we have their tensor product $U \otimes V$, defined by the representation map $b \mapsto b_{U} \otimes b_{V} .{ }^{8}$ Note that $1 \in A$ need not be represented by $1_{U} \otimes 1_{V}$. Thus the tensor product of two unital modules may be nonunital. ${ }^{9}$ A module $V$ is called semisimple when the unital submodule $V_{u}$ is semisimple in the usual sense. This is the case if and only if the representation map of $V$ vanishes on $B_{1}$. We call $A$-modules $U$ and $V$ equivalent, when there exist semisimple modules $S$ and $T$ such that $U \oplus S \simeq V \oplus T$. The set $\mathcal{M}(A)$ of equivalence classes of $A$-modules is a commutative semiring (possibly without unit element), where addition and multiplication are induced by direct sum and tensor product, respectively.

Degeneration of modules is defined using the closure of the conjugacy class of the representation map (see [12] II.3, [5], [6]). We write $U \unlhd V$ to indicate that $U$ is a degeneration of $V$. For $p, q \in \mathcal{M}(A)$ we define

$$
p \unlhd q: \Longleftrightarrow \exists U \in p, V \in q \quad U \unlhd V .
$$


Then $\unlhd$ is a partial order ${ }^{10}$ in $\mathcal{M}(A)$ compatible with addition and multiplication and such that $0 \unlhd p$ for all $p \in \mathcal{M}(A)^{11}$.

Asymptotic degeneration is defined by

$$
p \unlhd q: \Longleftrightarrow \exists k \in \mathbb{N} \forall N \gg 1 \quad p^{N} \unlhd N^{k} q^{N} .
$$

It is easy to see that $\gtrsim$ is a preorder in $\mathcal{M}(A)$ compatible with multiplication. It is also compatible with addition, as follows from Theorem 2 below.

We are going to characterize $\triangleleft$ in a simple and effective way. Before doing this we consider yet another order: Let $U, V$ be $A$-modules. We say that $U$ is a restriction of $V$, and write $U \leq V$, when $U$ is a quotient of a submodule of $V$. We define restriction and asymptotic restriction in $\mathcal{M}(A)$ by

$$
p \leq q \quad: \Longleftrightarrow \exists U \in p, V \in q \quad U \leq V
$$

and

$$
p \lesssim q: \Longleftrightarrow \exists k \in \mathbb{N} \forall N \gg 1 \quad p^{N} \leq N^{k} q^{N},
$$

respectively. It is easy to see that $\leq$ is a preorder in $\mathcal{M}(A)$, compatible with addition and multiplication, and that $\leq$ implies $\unlhd^{12}$. Hence $\leq$ is a partial order. It follows that $\lesssim$ is a preorder in $\mathcal{M}(\bar{A})$ compatible with multiplication (and by Theorem 2 also with addition), and that $\lesssim$ implies $\underset{\sim}{~}$

Theorem 2. Let $A$ ba a finite dimensional $\mathbb{C}$-algebra with a multiplicative basis $B$ such that $\operatorname{rad} A=\operatorname{lin} B_{1}$, where $B_{1} \subset B$. Take $p, q \in \mathcal{M}(A)$ and $U \in p, V \in q$. The following three conditions are equivalent:

$$
\begin{aligned}
& p \lesssim q, \\
& p \triangleleft q, \\
& \left.\begin{array}{rl}
\forall b & \in B_{1} \quad R\left(b_{U}\right) \leq R\left(b_{V}\right) \\
\forall b, c \in B_{1}, \theta & \in \mathbb{C} \quad b_{V}=\theta c_{V} \Longrightarrow b_{U}=\theta c_{U} .
\end{array}\right\}
\end{aligned}
$$

Theorem 2 will be proved in Section 3. We remark that the theorem also holds when the right hand sides of (2) and (3) are replaced by the apparently weaker conditions

$$
\forall \varepsilon>0 \forall N \gg 1 \quad p^{N} \unlhd 2^{\lfloor\varepsilon N\rfloor} q^{N}
$$

and

$$
\forall \varepsilon>0 \quad \forall N \gg 1 \quad p^{N} \leq 2^{\lfloor\varepsilon N\rfloor} q^{N},
$$

respectively. (This will follow from the proof of Theorem 2.) Hence these relaxations have no effect on the resulting notions of asymptotic degeneration and asymptotic restriction. 


\section{Proofs}

For $p, q \in \mathcal{M}(A)$ and $U \in p, V \in q$ we have

$$
p \unlhd q \Longleftrightarrow \exists k \forall N \gg 1 \exists S, T \quad S \oplus U^{\otimes N} \unlhd T \oplus \bigoplus_{1}^{N^{k}} V^{\otimes N},
$$

and

$$
p \lesssim q \Longleftrightarrow \exists k \forall N \gg 1 \exists T \quad U^{\otimes N} \leq T \oplus \bigoplus_{1}^{N^{k}} V^{\otimes N}
$$

where it is understood that $k, N$ are positive integers and $S, T$ semisimple modules. First we prove three lemmas.

Lemma 1. Let $A$ be a finite dimensional $\mathbb{C}$-algebra and let $U, V$ be $A$-modules. Then the following conditions are equivalent:

$$
\exists r \in \mathbb{N}, T \text { semisimple } \quad U \leq T \oplus \bigoplus_{1}^{r} V
$$

$$
\text { ann } V \cap \operatorname{rad} A \subset \operatorname{ann} U \text {. }
$$

When the conditions are satisfied one may choose $r \leq \operatorname{dim} U \cdot \operatorname{dim} A$.

The estimate of $r$ is easily improved, but we will not need this. ${ }^{13}$

Proof. Suppose that (9) holds. Since $\operatorname{rad} A \subset \operatorname{ann} T$ and since the annihilator increases when a module is being replaced by a submodule or a quotient we have

$$
\operatorname{ann} V \cap \operatorname{rad} A=\operatorname{ann}\left(T \oplus \bigoplus_{1}^{r} V\right) \cap \operatorname{rad} A \subset \operatorname{ann} U \cap \operatorname{rad} A \subset \operatorname{ann} U
$$

Conversely, suppose that (10) holds. Take a basis $\left(x_{1}, \ldots, x_{m}\right)$ of $U$ and elements $y_{1}, \ldots, y_{n}$ of $V$ such that ann $V=\bigcap_{i=1}^{n} \operatorname{ann}\left(y_{i}\right)$ and $n \leq \operatorname{dim} A$. This is possible since we may choose the $y_{j}$ inductively with strictly decreasing $\operatorname{dim} \bigcap_{i=1}^{j} \operatorname{ann}\left(y_{i}\right)$. Define

$$
y:=y_{1} \oplus \ldots \oplus y_{n} \in \bigoplus_{1}^{n} V
$$

Then

$$
\operatorname{ann} V=\operatorname{ann}(y)
$$


and therefore

$$
\begin{aligned}
& U \leq \bigoplus_{1}^{m} A x_{i} \quad \text { [quotient } \\
& \simeq \bigoplus_{1}^{m} A / \operatorname{ann}\left(x_{i}\right) \\
& \leq \bigoplus_{1}^{m} A / \operatorname{ann} U \quad \text { [quotient } \\
& \leq \bigoplus_{1}^{m} A /(\operatorname{ann} V \cap \operatorname{rad} A) \quad\lceil\text { quotient, by }(10)\rfloor \\
& \left.\leq \bigoplus_{1}^{m}(A / \operatorname{ann} V \oplus A / \operatorname{rad} A) \quad \text { [submodule }\right\rfloor \\
& \simeq \bigoplus_{1}^{m} A / \operatorname{ann}(y) \oplus \bigoplus_{1}^{m} A / \operatorname{rad} A \\
& \simeq \bigoplus_{1}^{m} A y \oplus \bigoplus_{1}^{m} A / \operatorname{rad} A \\
& \left.\leq \bigoplus_{1}^{m} \bigoplus_{1}^{n} V \oplus \bigoplus_{1}^{m} A / \operatorname{rad} A \quad \text { 「submodule }\right\rfloor .
\end{aligned}
$$

Since $\bigoplus_{1}^{m} A / \operatorname{rad} A$ is semisimple, this implies (9) with $r=m \cdot n \leq \operatorname{dim} U \cdot \operatorname{dim} A$.

The next lemma is well known.

Lemma 2. Suppose that $E$ is a finite dimensional complex vector space and that $z_{1}, \ldots, z_{q} \in E$ are pairwise linearly independent nonzero vectors. Then for sufficiently large $M$ the vectors $z_{1}^{\otimes M}, \ldots, z_{q}^{\otimes M} \in E^{\otimes M}$ are linearly independent.

Proof. By symmetry it suffices to show that for sufficiently large $M$ there is a linear form $f \in\left(E^{\otimes M}\right)^{*}$ such that

$$
f\left(z_{1}^{\otimes M}\right) \neq 0, \quad \forall i>1 \quad f\left(z_{i}^{\otimes M}\right)=0 .
$$

Polarization implies that this is equivalent to the existence of a homogeneous polynomial function $F$ on $E$ of degree $M$ with the property

$$
F\left(z_{1}\right) \neq 0, \quad \forall i>1 \quad F\left(z_{i}\right)=0 .
$$

The existence of such a form follows from the fact that $\left\{\left[z_{2}\right], \ldots,\left[z_{q}\right]\right\}$ is a Zariski closed subset of the projective space $\mathbf{P}(E)$ not containing $\left[z_{1}\right]$. 
Lemma 3. Let $A$ be a finite dimensional $\mathbb{C}$-algebra with a multiplicative basis $B$, where $\operatorname{rad} A=\operatorname{lin} B_{1}$, and let $V$ be an $A$-module. For sufficiently large $M \in \mathbb{N}$ the following is true: If $N$ is a positive integer and $W$ is isomorphic to a submodule of $V^{\otimes N}$ then $\operatorname{ann}\left(V^{\otimes M} \otimes W\right) \cap \operatorname{rad} A$ is linearly generated by $\operatorname{ann}(W) \cap B_{1}$ together with all $b-\theta^{M+N} c$ such that $b, c \in B_{1}$ and $b-\theta c \in$ ann $V$.

Proof. Clearly $\operatorname{ann}(W) \cap B_{1} \subset \operatorname{ann}\left(V^{\otimes M} \otimes W\right) \cap \operatorname{rad} A$. Since $V^{\otimes M} \otimes W$ is isomorphic to a submodule of $V^{\otimes(M+N)}$, the elements $b-\theta^{M+N} c$ with $b, c \in B_{1}$ and $b-\theta c \in$ ann $V$ also belong to $\operatorname{ann}\left(V^{\otimes M} \otimes W\right) \cap \operatorname{rad} A$. $\left\lceil\left(b-\theta^{M+N} c\right)_{V \otimes(M+N)}=\right.$ $\left.b_{V \otimes(M+N)}-\theta^{M+N} c_{V \otimes(M+N)}=\left(b_{V}\right)^{\otimes(M+N)}-\left(\theta c_{V}\right)^{\otimes(M+N)}=0.\right\rfloor$ This holds for arbitrary $M$.

Now take $M$ so large as to ensure the conclusion of Lemma 2 for $E=$ End $V$ and for every list $z_{1}, \ldots, z_{q}$ of pairwise linearly independent elements from $\left\{b_{V}\right.$ : $\left.b \in B_{1}\right\}$. Let $L$ denote the linear hull of $\operatorname{ann}(W) \cap B_{1}$ together with all $b-\theta^{M+N} c$ such that $b, c \in B_{1}$ and $b-\theta c \in$ ann $V$. We have just seen that

$$
L \subset \operatorname{ann}\left(V^{\otimes M} \otimes W\right) \cap \operatorname{rad} A
$$

and we have to prove equality. By way of contradiction let $\sum_{i=1}^{s} \alpha_{i} b_{i} \in \operatorname{ann}\left(V^{\otimes M_{\otimes}}\right.$ $W) \cap \operatorname{rad} A, \sum_{i=1}^{s} \alpha_{i} b_{i} \notin L$ be a counterexample of minimal length $s$ (where $\alpha_{i} \in \mathbb{C}$ and $\left.b_{i} \in B_{1}\right)$. Clearly $s>0$, and by the minimality of $s$ we have $\alpha_{i} \neq 0$ and $b_{i} \notin$ ann $W$. Moreover, the $\left(b_{i}\right)_{V}$ are pairwise linearly independent: Otherwise $\left(b_{1}\right)_{V}=\theta\left(b_{2}\right)_{V}$, say, so that $b_{1}-\theta b_{2} \in$ ann $V$ and therefore $b_{1}-\theta^{M+N} b_{2} \in L$. But then $\alpha_{1} b_{1}+\alpha_{2} b_{2}$ may be replaced by $\alpha_{1} b_{1}+\alpha_{2} b_{2}-\alpha_{1}\left(b_{1}-\theta^{M+N} b_{2}\right)=$ $\left(\alpha_{1} \theta^{M+N}+\alpha_{2}\right) b_{2}$, contradicting the minimality of $s$. Thus by the choice of $M$ the endomorphisms $\left(b_{i}\right)_{V \otimes M}=\left(\left(b_{i}\right)_{V}\right)^{\otimes M}$ are linearly independent.

Since $b_{i} \notin$ ann $W$, there is a linear form $y \in(\operatorname{End}(W))^{*}$ such that $\beta_{i}:=$ $y\left(\left(b_{i}\right)_{W}\right) \neq 0$ for all $i$. Now $\sum_{i=1}^{s} \alpha_{i} b_{i} \in \operatorname{ann}\left(V^{\otimes M} \otimes W\right)$, which means that $\sum_{i=1}^{s} \alpha_{i} \cdot\left(b_{i}\right)_{V \otimes M} \otimes\left(b_{i}\right)_{W}=0$. Applying $\mathrm{id}_{V \otimes M} \otimes y$ to this equation we get $\sum_{i=1}^{s} \alpha_{i} \beta_{i}\left(b_{i}\right)_{V \otimes M}=0$. But the $\left(b_{i}\right)_{V \otimes M}$ are linearly independent, hence all $\alpha_{i} \beta_{i}=$ 0 . This is a contradiction, since $s>0, \alpha_{i} \neq 0$ and $\beta_{i} \neq 0$.

Proof of Theorem 2. $(4) \Rightarrow(5)$ : This has already been observed just before of the statement of Theorem 2.

$(5) \Rightarrow(6)$ : In view of $(7)$ we have for some $k \in \mathbb{N}$ and for $N \gg 1$

$$
R\left(b_{U}\right)^{N}=R\left(b_{U \otimes N}\right) \leq N^{k} R\left(b_{V \otimes N}\right)=N^{k} R\left(b_{V}\right)^{N},
$$

since the rank decreases under degeneration and $b$ annihilates $T$. Taking $N$-th roots and letting $N$ grow to infinity we obtain $R\left(b_{U}\right) \leq R\left(b_{V}\right)$. We also have for $N \gg 1$

$$
b_{V}=\theta c_{V} \Longrightarrow b_{V \otimes N}=\theta^{N} c_{V^{\otimes N}} \Longrightarrow b_{U \otimes N}=\theta^{N} c_{U \otimes N} \Longrightarrow\left(b_{U}\right)^{\otimes N}=\theta^{N}\left(c_{U}\right)^{\otimes N}
$$


since the annihilator of modules increases under degeneration and $b$ and $c$ annihilate $S$. We assume without loss of generality $b_{U} \neq 0$ and $c_{U} \neq 0$ (hence $\theta \neq 0$ ) and choose $y \in\left(\operatorname{End}_{\mathbb{C}} U\right)^{*}$ such that $y\left(b_{U}\right)=\beta \neq 0, y\left(c_{U}\right)=\gamma \neq 0$. Applying $y^{\otimes N}$ to $\left(b_{U}\right)^{\otimes N}=\theta^{N}\left(c_{U}\right)^{\otimes N}$ and $y^{\otimes N} \otimes \operatorname{id}_{\operatorname{End}_{\mathbb{C}} U}$ to $\left(b_{U}\right)^{\otimes(N+1)}=\theta^{N+1}\left(c_{U}\right)^{\otimes(N+1)}$ we obtain $\beta^{N}=\theta^{N} \gamma^{N}$ and $\beta^{N} b_{U}=\theta^{N+1} \gamma^{N} c_{U}$. This implies $b_{U}=\theta c_{U}$.

$(6) \Rightarrow(4)$ : Set $m:=\operatorname{dim} U$ and $n:=\operatorname{dim} V$. For the moment we view $V$ simply as a vector space (rather than an $A$-module) and use some classical information on the decomposition of $V^{\otimes N}$ into simple $G L(V)$-modules, due to Frobenius, Young, Schur and Weyl (see Fulton-Harris [8], Theorem 6.3 and (4.11)):

$$
V^{\otimes N} \simeq \bigoplus_{\lambda \in P_{n}(N)} \bigoplus_{1}^{f_{\lambda}} S_{\lambda}(V)
$$

where $P_{n}(N)$ is the set of partitions of $N$ into at most $n$ parts, $S_{\lambda}(V)$ denotes a simple $G L(V)$-module with highest weight $\lambda=\left(\lambda_{1} \ldots, \lambda_{n}\right)$ and $f_{\lambda}$ is the dimension of the simple $S_{N}$-module corresponding to $\lambda$. We have

$$
\operatorname{dim} S_{\lambda}(V)=\prod_{1 \leq i<j \leq n} \frac{l_{i}-l_{j}}{j-i}
$$

where $l_{i}:=\lambda_{i}+n-i$, and

$$
f_{\lambda}=\frac{N ! \prod_{i<j}\left(l_{i}-l_{j}\right)}{l_{1} ! \cdot \ldots \cdot l_{n} !}
$$

The dimension of $S_{\lambda}(V)$ as well as the size of $P_{n}(N)$ are polynomially bounded in $N$ :

$$
\left|P_{n}(N)\right| \leq N^{n}, \quad \operatorname{dim} S_{\lambda}(V) \leq N^{n^{2}} .
$$

In contrast, $f_{\lambda}$ is typically of exponential size in $N$. We will not use this observation explicitly, but it is helpful for an understanding of the proof.

How does (11) relate to the $A$-module structure of $V^{\otimes N}$ ? Any $g \in G L(V)$ acts on $V^{\otimes N}$ as the tensor power $g^{\otimes N}$. By definition, $G L(V)$-submodules of $V^{\otimes N}$ are therefore stabilized by such $g^{\otimes N}$. Since $G L(V)$ is dense in $\operatorname{End}(V)$, a continuity argument shows that $G L(V)$-submodules of $V^{\otimes N}$ are also stabilized by tensor powers of arbitrary linear endomorphisms of $V$. In particular this is true for $b_{V \otimes N}=\left(b_{V}\right)^{\otimes N}$, where $b \in B$. Hence $G L(V)$-submodules of $V^{\otimes N}$ are automatically $A$-submodules. A similar continuity argument yields that isomorphic $G L(V)$-submodules of $V^{\otimes N}$ are also isomorphic as $A$-modules. By transport of structure the right hand side of (11) is an $A$-module and (11) is an isomorphism of $A$-modules. By what we have just seen the direct sum on the right hand side of (11) is a decomposition into $A$-submodules. (The newborn $A$-modules $S_{\lambda}(V)$ need not be simple or indecomposable.) 
Now fix $M$ such that the conclusion of Lemma 3 holds. In view of (8) it suffices to show

$$
\exists k \forall N \gg 1 \exists T \quad U^{\otimes(M+N)} \leq T \oplus \bigoplus_{1}^{N^{k}} V^{\otimes(M+N)},
$$

where $T$ is understood to be a semisimple $A$-module. Besides (11) and (13) we will need the isomorphism

$$
U^{\otimes(M+N)} \simeq \bigoplus_{\mu \in P_{m}(M+N)} \bigoplus_{1}^{f_{\mu}} S_{\mu}(U)
$$

analogous to (11) and the estimates

$$
\left|P_{m}(M+N)\right| \leq(M+N)^{m}, \quad \operatorname{dim} S_{\mu}(U) \leq(M+N)^{m^{2}}
$$

analogous to (13). In view of the $A$-module isomorphisms (11) and (15) we may reformulate (14) as

$$
\exists k \forall N \gg 1 \exists T \bigoplus_{\mu \in P_{m}(M+N)} \bigoplus_{1}^{f_{\mu}} S_{\mu}(U) \leq T \oplus \bigoplus_{1}^{N^{k}}\left(V^{\otimes M} \otimes \bigoplus_{\lambda \in P_{n}(N)} \bigoplus_{1}^{f_{\lambda}} S_{\lambda}(V)\right)
$$

By (16) this is a consequence of

$$
\begin{aligned}
\exists k \forall N \gg 1 \forall \mu \in P_{m}(M+N) \exists T & \bigoplus_{1}^{f_{\mu}} S_{\mu}(U) \\
\leq & T \oplus \bigoplus_{1}^{N^{k}}\left(V^{\otimes M} \otimes \bigoplus_{\lambda \in P_{n}(N)} \bigoplus_{1}^{f_{\lambda}} S_{\lambda}(V)\right)
\end{aligned}
$$

with the same stipulation for $T$. This in turn is implied by

$\exists k, l \forall N \gg 1 \forall \mu \in P_{m}(M+N) \exists T \quad S_{\mu}(U) \leq T \oplus \bigoplus_{1}^{N^{k}}\left(V^{\otimes M} \otimes \bigoplus_{\substack{\lambda \in P_{n}(N) \\ N^{l} f_{\lambda} \geq f_{\mu}}} S_{\lambda}(V)\right)$.

「Use $\bigoplus_{\lambda \in P_{n}(N), N^{l} f_{\lambda} \geq f_{\mu}} \bigoplus_{1}^{f_{\mu}} S_{\lambda}(V) \leq \bigoplus_{1}^{N^{l}} \bigoplus_{\lambda \in P_{n}(N)} \bigoplus_{1}^{f_{\lambda}} S_{\lambda}(V)$.」 Setting

$$
W=W_{l, N, \mu}:=\bigoplus_{\substack{\lambda \in P_{n}(N) \\ N^{l} f_{\lambda} \geq f_{\mu}}} S_{\lambda}(V)
$$


it suffices to show

$$
\exists l \forall N \gg 1 \forall \mu \in P_{m}(M+N) \quad \operatorname{ann}\left(V^{\otimes M} \otimes W\right) \cap \operatorname{rad} A \subset \operatorname{ann} S_{\mu}(U) .
$$

「Lemma 1 then yields

$$
\exists r, T \quad S_{\mu}(U) \leq T \oplus \bigoplus_{1}^{r}\left(V^{\otimes M} \otimes W\right)
$$

with

$$
r \leq \operatorname{dim} S_{\mu}(U) \cdot \operatorname{dim} A \leq(M+N)^{m^{2}} \operatorname{dim} A
$$

by (16).」 We check the inclusion in (17) on the set of linear generators of the left hand side as given by Lemma 3 (which is applicable by our choice of $M$ ).

First consider a generator of the form $b-\theta^{M+N} c$, where $b, c \in B_{1}$ and $b-$ $\theta c \in$ ann $V$. By $(6)$ we have $b-\theta c \in$ ann $U$, hence $b-\theta^{M+N} c \in \operatorname{ann} U^{\otimes(M+N)}$ and therefore $b-\theta^{M+N} c \in$ ann $S_{\mu}(U)$. Next consider a generator of the form $b \in \operatorname{ann}(W) \cap B_{1}$. For any $\mu$ we have

$$
V^{\otimes N} \simeq \bigoplus_{\lambda \in P_{n}(N)} \bigoplus_{1}^{f_{\lambda}} S_{\lambda}(V)=X \oplus Y
$$

where

$$
X:=\bigoplus_{\substack{\lambda \in P_{n}(N) \\ N^{l} f_{\lambda} \geq f_{\mu}}} \bigoplus_{1}^{f_{\lambda}} S_{\lambda}(V), \quad Y:=\bigoplus_{\substack{\lambda \in P_{n}(N) \\ N^{l} f_{\lambda}<f_{\mu}}} \bigoplus_{1}^{f_{\lambda}} S_{\lambda}(V) .
$$

Since $b$ annihilates $W$ and since $X$ and $W$ are composed of the same $A$-modules $S_{\lambda}(V)$ (with possibly different positive multiplicities), $b$ also annihilates $X$ and we conclude from (13) and the definition of $Y$

$$
R\left(b_{V}\right)^{N}=R\left(b_{V}^{\otimes N}\right)=R\left(b_{V \otimes N}\right) \leq \operatorname{dim} Y \leq N^{-l} f_{\mu} N^{n^{2}+n} .
$$

If (arguing by contradiction) $b \notin$ ann $S_{\mu}(U)$ for some $\mu$, we may estimate

$$
R\left(b_{U}\right)^{M+N}=R\left(b_{U \otimes(M+N)}\right) \geq R\left(b_{\Theta_{1}^{f_{\mu}} S_{\mu}(U)}\right) \geq f_{\mu}
$$

By assumption (6) we have $R\left(b_{U}\right) \leq R\left(b_{V}\right)$. Together with (18) and (19) this gives

$$
f_{\mu} \leq\left(N^{-l} f_{\mu} N^{n^{2}+n}\right)^{1+M / N}
$$

In view of the trivial estimate $f_{\mu} \leq \operatorname{dim} U^{\otimes(M+N)} \leq m^{M+N}$ this is impossible for $l>n^{2}+n$ and large $N$. This proves (17) and the theorem. 
Proof of Theorem 1. Both $U$ and $V$ are $\nu$-nilpotent for some positive integer $\nu$ in the sense that $U(w)=V(w)=0$ for all paths $w$ of length $\geq \nu$. Without loss of generality the semiring $\mathcal{N}(Q)$ in the statement of the theorem may therefore be replaced by the subsemiring $\mathcal{N}_{\nu}(Q)$ of all $\nu$-nilpotent equivalence classes of $Q$-modules. A well known equivalence of categories ([2], Theorem 1.5) induces an isomorphism of semirings $\mathcal{N}_{\nu}(Q) \rightarrow \mathcal{M}(A)$, where $A$ is the path algebra of $Q$ divided by the ideal spanned by all paths of length at least $\nu$, and with the set of residue classes of all paths of length at most $\nu-1$ as a multiplicative basis. (The radical of $A$ is spanned by the residue classes of paths of positive length. Tensor products of $\nu$-nilpotent $Q$-modules and of $A$-modules correspond up to equivalence.) This isomorphism respects degeneration (Bongartz [4]) and therefore asymptotic degeneration. Now Theorem 1 follows from Theorem 2.

\section{Acknowledgement}

I thank K. Bongartz, H. Kraft and M. Nüsken for having called my attention to some relevant literature, and Matthias Franz and the referee for carefully reading the manuscript.

\section{Annotations}

1. Bongartz [6] uses a kind of inverse notation, that does not suit the purposes of the present paper.

2. Code equivalence classes by suitable representatives.

3. This is similar to the definition of the tensor product for representations of finite groups.

4. A warning: By an example of J. Carlson (Riedtmann [15], p. 282) there are $p, q \in \mathcal{M}(Q)$ and $U \in p, V \in q$ such that $p \unlhd q, U$ and $V$ have the same dimension vector, but $U$ is not a degeneration of $V$.

5. Degenerate $V \in q$ along a Jordan-Hölder-series to a semisimple module $p$ (see [13], Proposition 4.3) and observe that $p$ is nilpotent, since $q$ is. Hence $p$ is trivial.

The property $0 \unlhd V$ is actually characteristic for nilpotent modules: Suppose $0 \unlhd V$. By the Hilbert Criterion (see Kraft [13], III,2) $V$ degenerates to zero along a one parameter subgroup of the 'group of coordinate changes' $\prod_{\alpha \in Q_{0}} G L_{n_{\alpha}}$, where we assume $V(\alpha)=\mathbb{C}^{n_{\alpha}}$. Without loss of generality (replace $V$ by an isomorphic copy) the one parameter subgroup is diagonal in every $G L_{n_{\alpha}}$. Then $V(l)$ is strictly upper triangular for any closed (directed) path $l$. This implies that $V$ is nilpotent.

6. This follows from three observations: 1 . An equivalence class is specified by a numerical partition, interpreted as the sequence of sizes of nontrivial nilpotent Jordan blocks arranged in nonincreasing order. 2. A single nilpotent Jordan 
block of size $m \geq 2$ is mapped to the function $f_{m}: j \mapsto \min \{m-j, 0\}$. 3. Any convex function $f: \mathbb{Z}_{>0} \rightarrow \mathbb{Z}_{\geq 0}$ with finite support is a unique linear combination of such $f_{m}$ with nonnegative integral coefficients.

7. Note that the image of $\varphi$ separates points.

8. The tensor product depends on the multiplicative basis chosen, as one sees for example by looking at group algebras of cyclic groups, which have two distinguished multiplicative bases, related by the Fourier Transform.

9. This is the reason for allowing nonunital modules.

10. Antisymmetry: As has first been observed by Gabriel [10] (see also Kraft [12], II,4), the Jordan-Hölder multiplicities of modules are preserved under degeneration. This holds in the non-unital case as well. Now assume $p \unlhd q$ and $q \unlhd p$ and choose $U \in p$ and $V \in q$. Then $U \oplus S \unlhd V \oplus T$ and $V \oplus T^{\prime} \unlhd U \oplus S^{\prime}$ for certain semisimple modules $S, S^{\prime}, T, T^{\prime}$. Without loss of generality $S=S^{\prime}$. Then $V \oplus T$ and $V \oplus T^{\prime}$ have the same Jordan-Hölder multiplicities, hence $T=T^{\prime}$, hence $U \oplus S \simeq V \oplus T$.

11. Degenerate $U \in p$ along a Jordan-Hölder series as in Kraft [12], II,4.

12. If $U \in p, V \in q, W \in r$ and if $0 \rightarrow U \rightarrow V \rightarrow W \rightarrow 0$ is exact, then $p+r \unlhd q$ and therefore $p \unlhd q$ and $r \unlhd q$.

13. The lemma implies that for $p, q \in \mathcal{M}(A)$ the following conditions are equivalent:

$\exists r \in \mathbb{N} \quad p \leq r \cdot q$

$\exists r \in \mathbb{N} \quad p \unlhd r \cdot q$

(iii) $\quad \operatorname{ann} V \cap \operatorname{rad} A \subset \operatorname{ann} U$ for some (and hence for all) $U \in p, V \in q$.

\section{References}

[1] Abeasis, S., Del Fra, A., Degenerations for the representations of a quiver of type $A_{m}, J$. Algebra 93 (1985), 376-412.

[2] Auslander, M., Reiten, I., Smalø, O., Representation Theory of Artin Algebras, Cambridge Studies in Advanced Mathematics 36 (1995).

[3] Bernstein, I. N., Gelfand, I. M., Ponomarev, V. A., Coxeter functors and a theorem of Gabriel, Uspechi Mat. Nauk 28 (1973), 19-33.

[4] Bongartz, K., A geometric version of the Morita equivalence, J. Algebra 139 (1991), 159179.

[5] Bongartz, K., Degenerations for representations of tame quivers, Ann. Sci. Ecole Norm. Sup. 28 (1995), 647-668.

[6] Bongartz, K., Some geometric aspects of representation theory, in: Algebras and Modules I, CMS conference proceedings vol. 23, ed. by Reiten, I., Smal $\varnothing$, S. O. and Solborg, $\varnothing$. (1998), p. 1-27.

[7] Dlab, V., Ringel, C. M., Indecomposable representations of graphs and algebras, Memoirs Amer. Math. Soc. 173 (1976).

[8] Fulton, W., Harris, J., Representation Theory, Graduate Texts in Mathematics 129, Springer-Verlag, 1991.

[9] Gabriel, P., Unzerlegbare Darstellungen I, Man. Math. 6 (1972), 71-103.

[10] Gabriel, P., Finite representation type is open, Springer Lecture Notes 488 (1975), 132-155.

[11] Gerstenhaber, M, On nilalgebras and linear varieties of nilpotent matrices III, Ann. Math. 70, No. 1 (1959). 
[12] Kraft, H., Geometric Methods in Representation Theory, Springer Lecture Notes 944 (1982), 180-258.

[13] Kraft, H., Geometrische Methoden in der Invariantentheorie, Vieweg, 1984.

[14] Kraft, H., Procesi, C., Closures of conjugacy classes of matrices are normal, Invent. Math. 53 (1979), 227-247.

[15] Riedtmann, C., Degenerations for representations of quivers with relations, Ann. Sci. Ecole Norm. Sup. 4 (1986), 275-301.

[16] Riedtmann, C., Personal communication (1999/2000).

[17] Strassen, V., The asymptotic spectrum of tensors, J. reine angew. Math. 384 (1988), 102-152.

[18] Zwara, G., Degenerations of finite dimensional modules are given by extensions, to appear in Compositio Math.

Volker Strassen

Universität Konstanz

Fakultät für Mathematik und Informatik

Universitätsstr. 10

D-78457 Konstanz

Germany

e-mail: strassen@mathe.uni-konstanz.de

(Received: November 26, 1998) 